\title{
EDITORIALS
}

\section{Getting in Step: Electronic Health Records and their Role in Care Coordination}

\author{
David W. Bates, MD, MSc ${ }^{1,2,3}$ \\ 'Division of General Internal Medicine and Center of Excellence for Patient Safety Research and Practice Brigham and Women's Hospital, \\ Boston, MA, USA; ${ }^{2}$ Harvard Medical School, Boston, MA, USA; ${ }^{3}$ Department of Health Policy and Management Harvard School of Public \\ Health, Boston, MA, USA.
}

\author{
KEY WORDS: chronic diseases; meaningful use; health care policy; \\ health information technology. \\ $\mathrm{J}$ Gen Intern Med 25(3):174-6 \\ DOI: $10.1007 / \mathrm{s} 11606-010-1252-\mathrm{x}$ \\ (c) Society of General Internal Medicine 2010
}

$\mathrm{T}$ he U.S. is making a historic investment in federal support for health information technology, which will likely approach $\$ 50$ billion. ${ }^{1}$ Most of this investment will go out in the form of incentives to providers who adopt electronic health records (EHRs) both outside the hospital and inside it. The rationale for this policy change is the belief that EHR use will reduce the costs of care, and improve quality and safety.

While there is widespread belief this will occur, the evidence with respect to the impact of EHRs on costs and quality has been mixed. ${ }^{2}$ Much of the trial data come from "home-grown" electronic records. Models show that in these settings costs can be reduced substantially with EHRs across a range of assumptions, ${ }^{3}$ but real-world results are less certain especially with vendor-developed records. Regarding quality, while there is clear evidence that in specific circumstances EHRs do improve performance for some domains such as preventive care and use of medications when decision support is delivered, ${ }^{4}$ the evidence is much more mixed for other domains, and many commercial applications include relatively little if any decision support. Cross-sectional studies that have asked whether or not EHR use has been associated with improved quality performance in the ambulatory setting have mostly found that it does not-in one study, Linder found no systematic association between EHR use and better quality performance. ${ }^{5}$ In another, Zhou asked whether length of EHR use was associated with better performance on quality measures, and again found that it was not. ${ }^{6}$ A more hopeful result came from Friedberg et al. who found that frequently used multifunctional EHRs were associated with better performance on 5 of 13 HEDIS measures in Massachusetts. ${ }^{7}$ The key themes are that for care to improve, the electronic record needs to be reasonably robust, and it has to be used well.

If the costs of care are to be addressed, it will be absolutely essential to address the care of patients with chronic diseases, who account for approximately three quarters of all healthcare expenditures. ${ }^{8,9}$ However, it has been a challenge to use EHRs to improve care for this group. Most work has focused on the impact of clinical decision support and registries to improve

Published online February 2, 2010 care for these groups, and while there have been some modest successes for example for diabetes and coronary disease, ${ }^{10}$ the results have been decidedly mixed overall. ${ }^{4}$ In patients with chronic conditions, care coordination is especially pivotal, because it can reduce readmissions, and ensure that needed follow-up occurs.

The main care redesign approach-which is potentially transformative and is justifiably getting a lot of attention-is the patient-centered medical home. Although this concept was developed many years ago, ${ }^{11}$ it has not been widely implemented, largely because of the way we pay for care in this country. To improve quality, all practices need good care coordination, regardless of whether or not they are medical homes.

In this issue of JGIM, O'Malley et al. present arguably the most comprehensive assessment to date of the ability of the current iteration of vendor-developed EHRs to assist providers with care coordination. ${ }^{12}$ To do their assessment, the researchers used qualitative techniques, and performed 60 interviews in a national sample of practices using 17 different commercial EHRs.

They identified six key themes: 1) that EHRs needed to help with in-office communications-which they generally did adequately; 2) that they also needed to help with communication between clinicians and settings which was much less satisfactory; 3) that clinicians found information overflow a challenge; 4) that current records don't support care coordination planning; 5) that care coordination processes need to evolve; and 6) not surprisingly, that fee-for-service payment encourages billable event documentation, but not care coordination.

These issues have different solutions. The problem of between-clinician and between settings communication is being addressed by the development of clinical data exchanges between entities, and this is squarely within the sights of policy-makers. ${ }^{13}$ Even when such exchanges are developed, however, considerable challenges remain, for example how to make it easy for a provider to see immediately that a key piece of information like a cardiac catheterization from another site is present, or even more important that a result available from a discharge elsewhere needs early follow-up.

The problem of information overflow represents a fundamental informatics problem, and will require some redesign of current clinical systems. ${ }^{14}$ It should be possible to "strain out" much of the extraneous information, while highlighting the few items that are truly need to be addressed soon.

However, the themes around the observation that current EHRs do not support care coordination well, and that the 
overall process needs to be redesigned represent the central and most important part of the results, with the most profound implications. No EHR in this study could claim exemplary performance in this area, and there will be a tight linkage between performing good care coordination, implementing the patient-centered medical home, and actually delivering the results with respect to improvement that everyone wants to see. To move to team care, which is a central feature of the medical home, will require tools that enable the various members of the team to document progress for patients, agree on goals, and stay on the same page with respect to progress and who is responsible for specific items. Many have made the assumption that this documentation needs to be in the notes of the record, but this doesn't have to be the case. Another approach would be to place much of this information in a new location in the record. Regardless, it will need to be linked with clinical decision support and registry functions that make it easier for care coordinators and providers alike to readily track patients.

But as O'Malley et al. found, these tools simply do not exist today in most EHRs, which means they will need to be developed. Even the underlying processes in practices in these areas are likely to evolve substantially in the coming years. This should be a key area of attention for SGIM members in the next several years, since it is especially important that this work well in primary care.

This work also illustrates the enormous value of qualitative research in evaluating healthcare information technology. Increasingly, mixed-model approaches should be used in informatics, even when the main outcomes are quantitative, because they can help elucidate what did and did not work. When addressing an area like this one which has received little previous attention, qualitative approaches are especially useful.

These findings have a number of implications. Today's commercially available EHRs do not come close to meeting provider needs with respect to care coordination, even though this is one of the most important domains for managing patients with chronic diseases. But practices themselves do not have well-developed processes for this area so this does not represent functionality the vendors can simply "add on." This implies that it is too early from the policy perspective to require tools such as this for certification of records, except at a very basic level. Instead the key approach in the near term should be to support research to develop and refine the needed tools.

In addition, there are some issues that can be readily addressed by vendors in the near term. For example, vendors should be representing data in standard ways to enable data exchange among users of different systems, and developing interfaces and tools that help address the data overload issue. A simple example of this latter issue is a tool that takes all the test results for a provider, puts them in a queue, and prioritizes them according to how abnormal they are. ${ }^{15}$ Some vendor applications do include tools like this today, but most do not. The current policy agenda in these areas should result in improvement.

The meaningful use definitions being developed should take the findings of this study into account, as should the Office of the National Coordinator. The current proposed meaningful use definitions around care coordination would require outpatient providers to participate in clinical data exchange and perform medication reconciliation at relevant encounters by 2011, and require providers to be able to receive electronic prescription fill data by 2013, while in 2015 providers would be asked to demonstrate a $10 \%$ decrease in the 30-day readmission rate and improvement on NQF-endorsed measures of care coordination. These criteria say nothing about care coordination within practices where most of it will take place, and thus it is not at all clear that meeting the 2011 and 2013 criteria will be sufficient to put practices in the position to be successful in 2015. The 2015 criteria appear to be relatively distant goalposts, and lots will need to change in the practices themselves and the EHRs they use if the 2015 criteria are to be met. While it may be reasonable for the criteria to be ends not means, there is a great deal to be done to address the issues of how to better coordinate care within practices. The Office of the National Coordinator and the Agency for Healthcare Research and Quality should begin supporting research in these areas immediately.

Ultimately, for improving care coordination to rise to the top of the list, payment reform will be essential. In the short run, this appears likely to take the form of accountable care organizations and bundling, which would be helpful with respect to aligning incentives, although more fundamental payment reform will likely eventually be necessary.

The current work by O'Malley et al. represents some of the best to date on how EHRs support care coordination, which again is a crucial function. Moving ahead will require more research in this area, as today's processes are immature and all of this will need to be interfaced with the medical home concept. Improving this could hardly be more important, as the patients who get the most benefit from care coordination account for a huge proportion of the costs in our healthcare system.

Acknowledgements: I thank Jennifer Love for her assistance with the preparation of this manuscript.

Corresponding Author: David W. Bates, MD, MSc; Division of General Internal Medicine and Center of Excellence for Patient Safety Research and Practice Brigham and Women's Hospital, Brigham Circle, 1620 Tremont St., 3rd Floor, Boston, MA 021201613, USA (e-mail: dbates@partners.org).

\section{REFERENCES}

1. American Recovery and Reinvestment Act of 2009; 2009

2. Chaudhry B, Wang $\mathbf{J}, \mathbf{W u} \mathbf{S}$, et al. Systematic review: impact of health information technology on quality, efficiency, and costs of medical care. Ann Intern Med. 2006; 144(10):742-52.

3. Wang SJ, Middleton B, Prosser LA, et al. A cost-benefit analysis of electronic medical records in primary care. Am J Med. 2003;114(5):397403.

4. Garg AX, Adhikari NK, McDonald H, et al. Effects of computerized clinical decision support systems on practitioner performance and patient outcomes: a systematic review. JAMA. 2005;293(10):1223-38.

5. Linder JA, Ma J, Bates DW, Middleton B, Stafford RS. Electronic health record use and the quality of ambulatory care in the United States. Arch Intern Med. 2007;167(13):1400-5.

6. Zhou L, Soran CS, Jenter CA, et al. The relationship between electronic health record use and quality of care over time. J Am Med Inform Assoc. 2009; 16(4):457-64.

7. Friedberg MW, Coltin KL, Safran DG, Dresser M, Zaslavsky AM, Schneider EC. Associations between structural capabilities of primary care practices and performance on selected quality measures. Ann Intern Med. 2009;151(7):456-63. 
8. Bodenheimer T, Wagner EH, Grumbach $\mathbf{K}$. Improving primary care for patients with chronic illness. JAMA. 2002;288(14):1775-9.

9. Hoffman C, Rice D, Sung HY. Persons with chronic conditions. Their prevalence and costs. JAMA. 1996;276(18):1473-9.

10. Sequist TD, Gandhi TK, Karson AS, et al. A randomized trial of electronic clinical reminders to improve quality of care for diabetes and coronary artery disease. J Am Med Inform Assoc. 2005;12(4): 431-7.

11. Homer CJ, Klatka K, Romm D, et al. A review of the evidence for the medical home for children with special health care needs. Pediatrics. 2008;122(4):e922-37.
12. O'Malley AS, Grossman JM, Cohen GR, Kemper NM, Pham HH. Are electronic medical records helpful for care coordination? Experiences of physician practices. J Gen Intern Med. XXXXXX 2010.

13. Adler-Milstein J, Bates DW, Jha AK. U.S. Regional health information organizations: progress and challenges. Health Aff (Millwood). 2009;28 (2):483-92.

14. Sittig DF, Wright A, Osheroff JA, et al. Grand challenges in clinical decision support. J Biomed Inform. 2008;41(2):387-92

15. Poon EG, Wang SJ, Gandhi TK, Bates DW, Kuperman GJ. Design and implementation of a comprehensive outpatient results manager. $\mathrm{J}$ Biomed Inform. 2003;36(1-2):80-91. 